\title{
Estimación del potencial energético del gas pobre a partir de la gasificación de cáscara de cacao y racimos de frutos vacíos de palma aceitera
}

\author{
César H. Zavala ${ }^{\star}$, Victor Pretell ${ }^{2}$, Javier Verastegui ${ }^{1}$ y Angel Ramirez ${ }^{3}$ \\ (1) Facultad de Ingeniería Mecánica, Universidad Nacional de Ingeniería, Rímac-Perú. \\ (Correo-e: czavalai@uni.edu.pe, javier.verastegui.|@uni.pe). \\ (2) Facultad de Ingeniería de Petróleo, Gas Natural y Petroquímica, Grupo de Investigación en Procesos Termoquímicos \\ y Energéticos, Universidad Nacional de Ingeniería, Rímac-Perú. (Correo-e: vpretell@fip.uni.edu.pe) \\ (3) Facultad de Ciencia, Laboratorio de Simulación e Investigación Numérica, Universidad Nacional de Ingeniería, \\ Rímac-Perú. (Correo-e: angelrg12481@gmail.com)
}

* Autor a quien debe ser dirigida la correspondencia.

Recibido Oct. 8, 2020; Aceptado Dic. 9, 2020; Versión final Ene. 18, 2021, Publicado Abr. 2021

\begin{abstract}
Resumen
El objetivo de esta investigación es estimar el potencial energético del gas pobre obtenido de los residuos agroindustriales de cacao y palma aceitera en el Perú usando la tecnología de gasificadores downdraft en base al modelo matemático de Serrato, pero aplicado a procesos de gasificación propuesto por el modelo de Roy. El modelo de Roy se ajustó con factores multiplicativos en las constantes de equilibrio y velocidad cinética para fragmentos de 3.3 a $5.5 \mathrm{~cm}$, esto se validó empleando datos experimentales obtenidos de la literatura. Se estima el máximo potencial energético del gas pobre de la cáscara de cacao y racimos de frutos vacíos de palma aceitera en el Perú de 4016.4 y 2108.7 TJ / año, respectivamente, para fragmentos de 3.3 $\mathrm{cm}$. Se concluye que la gasificación mediante gasificadores downdraft es una buena opción para generar bioenergía a partir de residuos de la agroindustria y satisfacer algunas carencias de energía eléctrica y térmica en zonas remotas del Perú.
\end{abstract}

Palabras clave: gasificación; potencial energético; gasificador downdraft; residuos agroindustriales

\section{Estimating producer gas energy potential from gasification of cocoa pod husk and oil palm empty fruit bunches}

\begin{abstract}
The objective of this research study is to estimate the energy potential of producer gas obtained from agroindustrial residues of cocoa and oil palm in Peru by using downdraft gasifier technology. This is performed based on the Serrato mathematical model, but applied to gasification processes proposed by Roy's model. The equilibrium constants and the kinetic velocity of the Roy's model are adjusted with multiplicative factors for fragments raging from 3.3 to $5.5 \mathrm{~cm}$. This is validated using experimental data obtained from the literature. The maximum energy potential of the producer gas from the cocoa pod husk and oil palm empty fruit in Peru is estimated to be 4016.4 and $2108.7 \mathrm{TJ} /$ year, respectively, for $3.3 \mathrm{~cm}$ fragments. It is concluded that gasification using downdraft gasifiers is a good option to generate agro-industrial waste bioenergy to solve electrical and thermal energy shortages in Peruvian remote areas.
\end{abstract}

Keywords: gasification; energy potential; downdraft gasifier; agro-industrial waste 


\section{INTRODUCCIÓN}

En las últimas dos décadas, el Perú ha crecido rápidamente en la producción de una variedad de cultivos agroindustriales para exportación y uso doméstico. Según el Balance de Energía del Perú 2017, un ejemplo de este crecimiento es la caña de azúcar, cuyo cultivo se encuentra muy extendido en la costa norte del Perú con el fin de obtener azúcar refinado, etanol combustible y bagazo. Este último subproducto tiene una participación significativa en la generación eléctrica nacional mediante tecnologías de combustión directa. Según la Serie de Estadísticas de Producción Agrícola (SEPA) del Perú, la producción de frutos de cacao y palma aceitera prácticamente se ha quintuplicado entre los años 2000 y 2007 (ver Tabla 1). Este importante crecimiento trae consigo la producción de grandes cantidades de residuos lignocelulósicos (Varnero et al., 2010), causando problemas de contaminación ambiental y de salud, como la propagación de enfermedades en los cultivos (Valadez et al., 2018). Según la SEPA, el cacao y la palma aceitera se cultivan en regiones de la sierra y selva del Perú, donde aún hay pobladores rurales que tienen necesidades de electricidad y calefacción.

La cáscara de cacao $(C D C)$ es el residuo más abundante del proceso de producción del cacao y representa el $75 \%$ del peso total de la fruta. Los productores lo consideran un subproducto indeseable y lo dejan descomponerse en el campo y según la información del Ministerio de Agricultura y Riego (MINAGRI) esos desechos de cacao se han utilizado mínimamente en la industria alimentaria. Considerando que en promedio cada tonelada de cacao en grano seco produce 10 toneladas de CDC (Valadez et al., 2018), se puede estimar que en el año 2017 se podrían haber producido 1.2 millones de toneladas de CDC.

Por otro lado, la palma aceitera es un prolífico productor de residuos de biomasa. El aceite representa solo el $10 \%$ de la producción de palma, siendo el resto diversos residuos de biomasa (Yiin et al., 2018). Según el MINAGRI cada hectárea de palma aceitera puede producir entre 50 y 70 toneladas de biomasa residual por año. Los tipos de residuos de biomasa producidos por la industria de la palma aceitera son racimos de frutos vacíos (RFV), fibras, cáscaras, cáscaras húmedas, semillas de palma, follaje y troncos; el $30.5 \%$ de esta biomasa residual está compuesta por RFV. Se puede estimar que la producción de racimos de frutos vacíos (RFV) es de 1.1 millones de toneladas por año. En consecuencia, existe un gran interés en el Perú por utilizar la biomasa agroindustrial de cacao y palma aceitera con fines energéticos; sin embargo, hasta la fecha ha habido muy poco uso comercial de los procesos de conversión de energía de CDC y RFV, por ejemplo, la tecnología de gasificación de biomasa por lecho fijo para producir gas pobre.

Cuando se utiliza la tecnología de los gasificadores de lecho fijo, la ceniza se convierte en un problema debido a la acumulación de escoria y el consiguiente ensuciamiento. Según los experimentos de Thengane et al. (2019) se ha demostrado que la ceniza se libera del carbón a temperaturas del orden de $1923^{\circ} \mathrm{K}$ (1650ㄷ), que están muy por encima de la temperatura de fusión de la mayoría de los minerales. Además, cuando el combustible se quema, esta ceniza se libera en forma viscosa, y una fracción de ella impacta y se adhiere a las paredes del gasificador, lo que, dada la enorme cantidad de biomasa involucrada en el proceso, puede interferir con la normalidad del funcionamiento del gasificador (Yan et al., 2016). Los compuestos gaseosos pueden ser combustibles $\left(\mathrm{C}_{\mathrm{x}} \mathrm{H}_{\mathrm{y}}, \mathrm{CO} \circ \mathrm{H}_{2}\right)$ e incombustibles $\left(\mathrm{CO}_{2}, \mathrm{SO}_{2}, \mathrm{NO}_{x}\right)$ (Hernández et al., 2004). Por lo tanto, se ha recomendado que el proceso de combustión en muchos diseños de gasificadores se opere muy por debajo de esta temperatura. Otra investigación indica que altas concentraciones de cenizas pueden tener efectos catalíticos adversos en los procesos de conversión termoquímica como la pirólisis, generando gases no condensables en detrimento de los productos líquidos (Asadieraghi et al., 2015). Por esta razón, se prefiere trabajar con combustibles que tengan valores bajos de contenido de cenizas. Los valores de ceniza en base seca encontrados para RFV varían de 5.36\% a 7\% (Asadieraghi, 2015; Yiin et al.,2018) y para CDC entre $6.99 \%$ y $13.5 \%$ (Tsai et al.,2018), que se consideran relativamente altos.

Muchos procesos de gasificación dependen principalmente de la composición de la biomasa residual, su contenido de humedad, la estequiometría de la reacción y el diseño del gasificador. Un modelo matemático adecuado para seguir los procesos en el gasificador de lecho fijo puede predecir los parámetros de rendimiento óptimos para su funcionamiento. Es posible considerar un proceso de gasificación real, a partir de un equilibrio químico en la zona de pirooxidación y reacciones químicas de velocidad finita controladas cinéticamente en la zona de reducción del gasificador (Giltrap, 2003; Babu, 2006; Sharma, 2008). La mayoría de los modelos cinéticos se basan en la ruta de reacción química prescrita por Wang (1993) para comprender mejor la conversión de carbón en la zona de reducción del gasificador.

Tabla 1: Producción y áreas cultivadas de las biomasas en los años 2000 y 2017.

\begin{tabular}{|c|c|c|c|c|}
\hline \multirow{2}{*}{ Cultivos } & \multicolumn{2}{|c|}{ Producción (t/año) } & \multicolumn{2}{c|}{ Área cultivada (ha/año) } \\
\cline { 2 - 5 } & 2000 & 2017 & 2000 & 2017 \\
\hline Cacao & 24786 & 1218140 & 41264 & 147304 \\
\hline Palma aceitera & 181155 & 852022 & 9990 & 58951 \\
\hline
\end{tabular}


Barman (2012), Jarungthammachote (2008) y Roy et al. (2009) validaron su modelo para predecir la composición del gas pobre con los datos experimentales de Jayah et al. (2003), pero solo para fragmentos de biomasa menores a $3.3 \mathrm{~cm}$ de diámetro. Cuando se aplica el modelo de Roy a los fragmentos de biomasa $(3.3,4.4$ y $5.5 \mathrm{~cm})$, la composición de los gases para diferentes tamaños de fragmentos presenta errores promedios predictivos que varían en el rango de $2.56 \%$ a $17.81 \%$. Además, la variación del error predictivo para la composición de $\mathrm{H}_{2}$ encontrada en fragmentos de 4.4 y $5.5 \mathrm{~cm}$ de diámetro osciló entre $33 \%$ y $49 \%$.

El objetivo de esta investigación es estimar el potencial energético del gas pobre obtenido a partir de la cáscara de cacao y racimos de frutos vacíos de palma aceitera en base al modelo matemático de Serrato (2016), pero aplicado a procesos de gasificación propuesto por el modelo de gasificación de Roy et al. (2009) basados en el equilibrio termodinámico en la zona de pirooxidación y la cinética de las reacciones en la zona de reducción. Las operaciones matemáticas no son complejas, aunque sí incluye un aceptable análisis fisicoquímico que lo hace adecuado para realizar un buen estudio para la optimización de gasificadores de lecho fijo. Se sabe que la gasificación de cualquier biomasa residual requiere un contenido de humedad muy bajo y un tamaño de fragmento muy pequeño de biomasa residual agrícola por temas de rendimiento y eficiencia (Torres et al., 2018). Sin embargo, en la práctica, es poco probable que estas condiciones ideales sean económicamente viables, por lo que se consideró necesario ampliar el rango de tamaño de partícula de biomasa. Con el fin de mejorar la predicción en la composición de los gases, se han introducido factores de multiplicación siguiendo el criterio de Barman (2012) y Jarungthammachote (2008), que permitieron obtener un rango de errores promedios predictivos para la composición de los gases más reducidos, variando desde $1.48 \%$ a $9.83 \%$ solamente. Asimismo, la variación del error predictivo para la composición de $\mathrm{H}_{2}$, en los fragmentos de 4.4 y $5.5 \mathrm{~cm}$ osciló entre $3.82 \%$ y $16.12 \%$; que es un rango mucho menor en comparación con el obtenido aplicando el modelo Roy original sin la introducción de factores multiplicadores.

\section{METODOLOGÍA}

Las muestras de racimos de palma aceitera de frutos vacíos (RFV) provienen de una plantación ubicada en la provincia de Lamas, Región de San Martín, Perú, y las cáscaras de cacao (CDC) de una plantación ubicada en el distrito de Polvora, provincia de Tocache, Región de San Martín, Perú. El análisis elemental se realizó según la norma ASTM D5373-Método A y ASTM D4239, los análisis de carbono, hidrógeno y nitrógeno (C, H, N) se llevaron a cabo, utilizando un analizador elemental modelo LECO 628, el análisis de azufre se realizó en el módulo 628 Series Sulfur Add-On y el contenido de oxígeno se obtuvo por diferencia.

El análisis próximo se realizó por termogravimetría según la norma ASTM D7582, utilizando un analizador termogravimétrico TGA 4000 de Perkin Elmer. Las muestras para todos los análisis se realizaron con un tamaño de partícula menor de $250 \mu \mathrm{m}$. Todas las muestras se mantuvieron en un desecador durante seis horas antes del análisis. El valor calorífico superior (HHV) se determinó de acuerdo a la norma ASTM D5865. Se quemó una muestra de 0,5 a 1,0 g de biomasa pulverizada menor de $850 \mu \mathrm{m}$ en una bomba calorimétrica adiabática Parr 1341 en condiciones controladas. Cada análisis se realizó por triplicado.

Para estimar el potencial energético del gas pobre de la biomasa ( $P E_{G P}, T J /$ año), se plantea la ecuación (1) teniendo como referencia al modelo matemático de Serrato (2016), pero en este caso lo aplicaremos a procesos de gasificación. Esta ecuación, consta de dos partes; la primera parte sirve para calcular la masa de residuos secos de la biomasa por año ( $\mathrm{M}_{\mathrm{rs}}$, $\mathrm{t} / \mathrm{año}$ ) y la segunda parte para calcular el máximo valor calorífico inferior que resulta óptimo por tonelada de gas pobre (LHVGP, TJ/t); este valor se obtiene utilizando el modelo de la gasificación ajustado, para fragmentos de biomasa entre 3.3 a $5.5 \mathrm{~cm}$ de diámetro.

$$
P E_{G P}=M_{r s} . L H V_{G P}
$$

La ecuación (2) establece la relación entre las variables más relevantes para estimar la masa de residuos secos ( $\left.\mathrm{M}_{\mathrm{rs}}\right)$ : el número de hectáreas correspondientes al área cultivada por año ( $\mathrm{S}$, ha/año), el rendimiento del cultivo expresado como el producto principal en toneladas por hectárea cultivada $\left(R_{c}, t / h a\right)$, el factor de residuo del cultivo como la relación en toneladas de residuos húmedos con respecto a las toneladas del producto principal $\left(\mathrm{F}_{\mathrm{r}}\right)$, y la fracción de residuos secos como la relación entre la masa de residuos húmedos y $\operatorname{secos}\left(Y_{r s}\right)$.

$$
M_{\mathrm{rs}}=\left(S \cdot R_{\mathrm{c}} \cdot \mathrm{F}_{\mathrm{r}} \cdot \mathrm{Y}_{\mathrm{rs}}\right)
$$

Tomando como referencia el gasificador de Jayah et al. (2003), Roy et al. (2009) divide el gasificador en dos zonas, y considera dos submodelos matemáticos. El primer submodelo es aplicado en la zona de pirooxidación, cuyas temperaturas son las más elevadas en el gasificador por lo que se aplica el modelo de equilibrio químico; el segundo submodelo es aplicado en la zona de gasificación o reducción, basada en el modelo cinético químico del "char" presentando un gran número de volúmenes de control elemental a lo largo de la zona reducción. En cada volumen de control se aplica el balance de masa y energía teniendo en cuenta 
las dimensiones del gasificador de Jayah et al. (2003). Las contribuciones de la ceniza en la biomasa y la pérdida de calor en el gasificador se han considerado en la ecuación de balance energético. La reacción global en la zona de oxidación de pirólisis se puede escribir como sigue:

$$
\mathrm{CH}_{m} \mathrm{O}_{\mathrm{n}}+\mathrm{wH}_{2} \mathrm{O}+\mathrm{aO} \mathrm{O}_{2}+3.76 \mathrm{aN}_{2} \rightarrow \mathrm{x}_{1} \mathrm{H}_{2}+\mathrm{x}_{2} \mathrm{CO}+\mathrm{x}_{3} \mathrm{CO}_{2}+\mathrm{x}_{4} \mathrm{H}_{2} \mathrm{O}+\mathrm{x}_{5} \mathrm{CH}_{4}+\mathrm{x}_{6} \mathrm{~N}_{2}+\mathrm{x}_{7} \mathrm{C}
$$

Los valores de $\mathrm{m}$ y $\mathrm{n}$ se calculan a partir del análisis elemental; el coeficiente a se obtiene de la relación de equivalencia (RE) que está definida como la relación de aire-biomasa real dividida entre la relación airebiomasa estequiométrica. El coeficiente $\mathrm{w}$, es la cantidad de agua que puede determinarse por el contenido de humedad de la biomasa (MC) y contenido de ceniza (ASH) de la siguiente manera:

$$
\mathrm{W}=\left(\frac{0.01 \mathrm{MC}}{(1-0.01 \mathrm{MC})(1-0.01 \mathrm{ASH})}\right)\left(\frac{\overline{\mathrm{M}}_{\mathrm{CH}_{\mathrm{m}} \mathrm{O}_{\mathrm{n}}}}{\overline{\mathrm{M}}_{\mathrm{H}_{2} \mathrm{O}}}\right)
$$

Las dos reacciones de equilibrio en la zona de pirooxidación son la reacción de cambio de gas-agua $\left(\mathrm{CO}_{(\mathrm{g})}+\mathrm{H}_{2} \mathrm{O}_{(\mathrm{l})} \leftrightarrow \mathrm{CO}_{2(\mathrm{~g})}+\mathrm{H}_{2(\mathrm{~g})}\right)$ y la reacción de metanización $\left(\mathrm{C}_{(\mathrm{s})}+2 \mathrm{H}_{2(\mathrm{~g})} \leftrightarrow \mathrm{CH}_{4(\mathrm{~g})}\right) ; y$ cuyas constantes de equilibrio son $\mathrm{K}_{1}$ y $\mathrm{K}_{2}$ respectivamente. Se realizan las ecuaciones de balance de masa para $\mathrm{C}, \mathrm{H}, \mathrm{O}$ y $\mathrm{N}$ según la ecuación (3), y se adiciona una ecuación más; el carbono fijo (FC) en base seca.

$$
\begin{aligned}
& \mathrm{K}_{1}=\frac{\mathrm{x}_{3} \cdot \mathrm{x}_{1}}{\mathrm{x}_{2} \cdot \mathrm{x}_{4}}=\mathrm{e}^{\left(-\mathrm{g}_{\mathrm{CO}_{2}}^{0}-\mathrm{g}_{\mathrm{H}_{2}}^{0}+\mathrm{g}_{\mathrm{CO}}^{0}+\mathrm{g}_{\mathrm{H}_{2} \mathrm{O}}^{0}\right) / \mathrm{RT}} \\
& \mathrm{K}_{2}=\frac{\mathrm{x}_{5}}{\mathrm{x}_{1}^{2}} \cdot \mathrm{n}_{\mathrm{t}}=\mathrm{e}^{\left(-\mathrm{g}_{\mathrm{CH}_{4}}^{0}+2 \mathrm{~g}_{\mathrm{H}_{2}}^{0}\right) / \mathrm{RT}} \\
& \mathrm{C}=\frac{\mathrm{FC}}{\left(\mathrm{x}_{5}+\mathrm{x}_{7}\right)}
\end{aligned}
$$

La pérdida de calor $Q$ tiene valor negativo en sentido termodinámico, y se define como:

$$
Q_{\text {perdida }}=m_{A S H} \cdot C_{p_{A S H}}\left(T-T_{O}\right)+\sum_{i=1}^{6} x_{i}\left(h_{f_{1}}^{o}+\int_{T_{0}}^{T} C_{p_{i}} d T\right)+x_{7} \cdot C_{p_{C H A R}}\left(T-T_{0}\right)-\left(h_{f_{b i o m a s a}}^{o}+a \int_{T_{o}}^{T_{a}} C_{p_{\mathrm{O}_{2}}} d T+3.76 a \int_{T_{0}}^{T_{a}} C_{p_{N_{2}}} d T+w \cdot h_{f_{H_{2} O}}\right)
$$

Las cuatro reacciones consideradas en la zona de reducción son la reacción de Boudouard $\left(\mathrm{C}_{(\mathrm{s})}+\mathrm{CO}_{2(\mathrm{~g})} \leftrightarrow 2 \mathrm{CO}_{(\mathrm{g})}\right)$, reacción de cambio gas-agua $\left(\mathrm{C}_{(\mathrm{s})}+\mathrm{H}_{2} \mathrm{O}_{(\mathrm{g})} \leftrightarrow \mathrm{CO}_{(\mathrm{g})}+\mathrm{H}_{2(\mathrm{~g})}\right)$, reacción de metanización $\left(\mathrm{C}_{(\mathrm{s})}+2 \mathrm{H}_{2(\mathrm{~g})} \leftrightarrow \mathrm{CH}_{4(\mathrm{~g})}\right)$ y reacción de reformado por vapor $\left(\mathrm{CH}_{4(\mathrm{~g})}+\mathrm{H}_{2} \mathrm{O}_{(\mathrm{g})} \leftrightarrow \mathrm{CO}_{(\mathrm{g})}+3 \mathrm{H}_{2(\mathrm{~g})}\right)$, cuyas velocidades de reacción son $\mathrm{r}_{\mathrm{R} 1}$, $\mathrm{r}_{\mathrm{R} 2}$, $\mathrm{r}_{\mathrm{R} 3}$ y $\mathrm{r}_{\mathrm{R} 4}$ respectivamente. Estas velocidades dependerán de las fracciones molares (y), temperatura en la zona de reducción y constante de equilibrio.

$$
\begin{aligned}
& r_{R 1}=3616 \cdot e^{\left(\frac{-77390}{R T}\right)} \cdot\left(y_{\mathrm{CO}_{2}}-\frac{y_{\mathrm{CO}}^{2}}{\mathrm{~K}_{\mathrm{R} 1}}\right) ; \text { siendo } \mathrm{K}_{\mathrm{R} 1}=\mathrm{e}^{\frac{\left(-2 \mathrm{~g}_{\mathrm{f}, T, \mathrm{CO}}^{\circ}+\mathrm{CO}_{\mathrm{T}, T, \mathrm{CO}_{2}}^{\circ}\right)}{\mathrm{RT}}}
\end{aligned}
$$

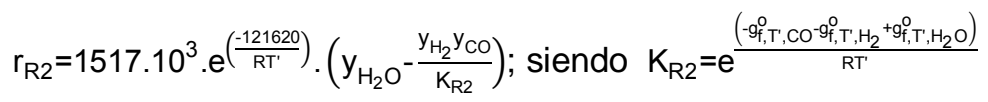

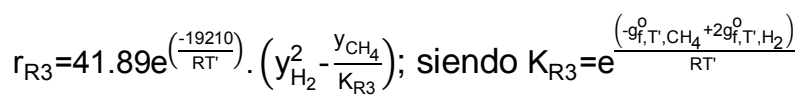

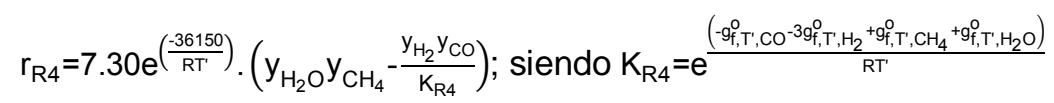

La pérdida de calor es despreciable en esta última zona y la temperatura de la composición del gas desciende hasta llegar a la salida de la zona de reducción. Según Waldheim (2001), el valor calorífico inferior (LHVGP) del gas pobre se determina a partir de la composición química del gas seco y el LHV de los componentes individuales. Los cálculos y gráficas pueden ser realizados utilizando el lenguaje de programación Python o Matlab.

$$
\operatorname{LHV}_{\mathrm{GP}}=0.01\left(12.622 \mathrm{y}_{\mathrm{CO}}+10.788 \mathrm{y}_{\mathrm{H}_{2}}+35.814 \mathrm{y}_{\mathrm{CH}_{4}}\right) ; \text { en } \mathrm{MJ} / \mathrm{m}^{3}
$$

\section{RESULTADO Y DISCUSIÓN}

En la Tabla 2, el análisis elemental, próximo y valores caloríficos de las muestras de RFV y CDC, son muy similares a los obtenidos en las referencias (Asadieraghi, 2015; Yiin et al., 2018; Tsai et al. ,2018). Tenemos valores elevados de materia volátil que facilitan la pirólisis de la biomasa. El contenido de carbono fijo (FC) es la masa sólida que queda después de la expulsión de la materia volátil (VM) de la biomasa, sin contar el contenido de cenizas (ASH) y humedad (MC); esta es la parte más resistente a la degradación térmica 
(Barman, 2012). El contenido de ASH de CDC es el doble de RFV por lo que se verá más afectado en el proceso de gasificación. El modelo de gasificación de Roy et al. (2009) ha sido útil porque aplica el término "pirólisis llameante" a la zona de pirooxidación del gasificador, que es la combustión de volátiles con un aporte inadecuado de aire por lo que los productos son principalmente $\mathrm{CO}$ e $\mathrm{H}_{2}$ en lugar de $\mathrm{CO}_{2}$ y $\mathrm{H}_{2} \mathrm{O}$.

Tabla 2: Análisis elemental, análisis próximo y valor calorífico de biomasa.

\begin{tabular}{|c|c|c|c|c|c|c|c|c|c|c|}
\hline \multirow{2}{*}{ Biomasa } & \multicolumn{4}{|c|}{$\begin{array}{c}\text { Análisis elemental } \\
(\%)\end{array}$} & \multicolumn{4}{c|}{$\begin{array}{c}\text { Análisis próximo } \\
(\%)\end{array}$} & $\begin{array}{c}\text { Valor calorífico } \\
(\mathrm{MJ} / \mathrm{kg})\end{array}$ \\
\cline { 2 - 12 } & $\mathrm{C}$ & $\mathrm{H}$ & $\mathrm{N}$ & $\mathrm{S}$ & $\mathrm{O}$ & $\mathrm{MC}$ & $\mathrm{ASH}$ & $\mathrm{VM}$ & $\mathrm{FC}$ & $\mathrm{HHV}$ \\
\hline RFV & 45.31 & 6.46 & 0.47 & 0.07 & 41.15 & 3.66 & 6.30 & 72.43 & 17.61 & 21.30 \\
\hline $\mathrm{CDC}$ & 42.0 & 5.9 & 0.83 & 0.12 & 36.6 & 4.35 & 13.92 & 65.31 & 16.42 & 18.85 \\
\hline
\end{tabular}

Tabla 3: Composición del gas considerando el modelo de Roy y el modelo ajustado en comparación con los datos experimentales de Jayah et al. (2003).

\begin{tabular}{|c|c|c|c|c|c|c|c|c|}
\hline $\begin{array}{l}\text { Diámetro de } \\
\text { fragmento } \\
\text { (cm) }\end{array}$ & $\begin{array}{c}\text { Prueba } \\
N^{\circ}\end{array}$ & Comparación & $\begin{array}{l}\mathrm{H}_{2} \\
(\%)\end{array}$ & $\begin{array}{l}C O \\
(\%)\end{array}$ & $\begin{array}{l}\mathrm{CO}_{2} \\
(\%)\end{array}$ & $\begin{array}{l}\mathrm{CH}_{4} \\
(\%)\end{array}$ & $\begin{array}{l}\mathrm{N}_{2} \\
(\%)\end{array}$ & $\begin{array}{c}\text { Error } \\
\text { promedio } \\
\text { predictivo } \\
(\%)\end{array}$ \\
\hline \multirow{15}{*}{3.3} & \multirow{5}{*}{1} & Jayah & 17.20 & 19.60 & 9.90 & 1.40 & 51.90 & \\
\hline & & Modelo Roy & 19.37 & 18.29 & 10.99 & 1.21 & 50.15 & \\
\hline & & Modelo ajustado & 19.30 & 18.30 & 10.99 & 1.24 & 50.16 & \\
\hline & & \% Error predictivo Roy & 12.60 & 6.69 & 11.02 & 13.85 & 3.38 & 9.51 \\
\hline & & $\%$ Error predictivo modificado & 12.20 & 6.61 & 11.05 & 11.12 & 3.36 & 8.87 \\
\hline & \multirow{5}{*}{2} & Jayah & 18.30 & 20.20 & 9.70 & 1.10 & 50.70 & \\
\hline & & Modelo Roy & 18.18 & 19.22 & 10.32 & 1.12 & 51.16 & \\
\hline & & Modelo ajustado & 18.10 & 19.24 & 10.32 & 1.17 & 51.17 & \\
\hline & & \% Error predictivo Roy & 0.65 & 4.87 & 6.42 & 1.80 & 0.91 & 2.93 \\
\hline & & \% Error predictivo modificado & 1.09 & 4.77 & 6.44 & 6.30 & 0.93 & 3.91 \\
\hline & \multirow{5}{*}{3} & Jayah & 17.20 & 19.40 & 9.70 & 1.10 & 52.6 & \\
\hline & & Modelo Roy & 17.44 & 19.66 & 10.03 & 1.04 & 51.82 & \\
\hline & & Modelo ajustado & 17.35 & 19.69 & 10.03 & 1.10 & 51.83 & \\
\hline & & \% Error predictivo Roy & 1.42 & 1.35 & 3.39 & 5.17 & 1.48 & 2.56 \\
\hline & & \% Error predictivo modificado & 0.87 & 1.48 & 3.41 & 0.20 & 1.46 & 1.48 \\
\hline \multirow{15}{*}{4.4} & \multirow{5}{*}{4} & Jayah & 17.00 & 18.40 & 10.60 & 1.30 & 52.70 & \\
\hline & & Modelo Roy & 19.83 & 19.07 & 10.56 & 1.20 & 49.33 & \\
\hline & & Modelo ajustado & 16.35 & 18.78 & 9.86 & 1.31 & 53.70 & \\
\hline & & \% Error predictivo Roy & 16.68 & 3.66 & 0.40 & 7.42 & 6.39 & 6.91 \\
\hline & & $\%$ Error predictivo modificado & 3.82 & 2.08 & 6.95 & 0.40 & 1.89 & 3.03 \\
\hline & \multirow{5}{*}{5} & Jayah & 13.20 & 19.70 & 10.80 & 1.30 & 55.00 & \\
\hline & & Modelo Roy & 18.46 & 19.20 & 10.32 & 1.15 & 50.86 & \\
\hline & & Modelo ajustado & 14.09 & 18.53 & 9.63 & 1.26 & 56.48 & \\
\hline & & \% Error predictivo Roy & 39.85 & 2.53 & 4.43 & 11.27 & 7.52 & 13.12 \\
\hline & & \% Error predictivo modificado & 6.74 & 5.93 & 10.79 & 3.00 & 2.70 & 5.83 \\
\hline & \multirow{5}{*}{6} & Jayah & 12.50 & 18.90 & 8.50 & 1.20 & 59.10 & \\
\hline & & Modelo Roy & 17.81 & 19.87 & 9.92 & 1.06 & 51.34 & \\
\hline & & Modelo ajustado & 11.98 & 19.23 & 8.85 & 1.15 & 58.78 & \\
\hline & & $\%$ Error predictivo Roy & 42.47 & 5.14 & 16.71 & 11.59 & 13.13 & 17.81 \\
\hline & & \% Error predictivo modificado & 4.16 & 1.77 & 4.15 & 3.86 & 0.54 & 2.90 \\
\hline \multirow{15}{*}{5.5} & \multirow{5}{*}{7} & Jayah & 15.50 & 19.10 & 11.40 & 1.10 & 52.90 & \\
\hline & & Modelo Roy & 20.57 & 19.34 & 10.47 & 1.23 & 48.39 & \\
\hline & & Modelo ajustado & 17.63 & 18.46 & 10.24 & 1.33 & 52.34 & \\
\hline & & \% Error predictivo Roy & 32.73 & 1.23 & 8.20 & 12.00 & 8.52 & 12.54 \\
\hline & & \% Error predictivo modificado & 13.77 & 3.34 & 10.19 & 20.69 & 1.06 & 9.81 \\
\hline & \multirow{5}{*}{8} & Jayah & 12.70 & 22.10 & 10.50 & 1.30 & 53.40 & \\
\hline & & Modelo Roy & 18.83 & 19.87 & 9.92 & 1.16 & 50.21 & \\
\hline & & Modelo ajustado & 14.75 & 18.45 & 9.74 & 1.26 & 55.80 & \\
\hline & & \% Error predictivo Roy & 48.24 & 10.07 & 5.48 & 10.69 & 5.97 & 16.09 \\
\hline & & $\%$ Error predictivo modificado & 16.12 & 16.53 & 7.26 & 2.77 & 4.50 & 9.43 \\
\hline & \multirow{5}{*}{9} & Jayah & 13.00 & 19.10 & 10.70 & 1.20 & 56.00 & \\
\hline & & Modelo Roy & 17.38 & 20.06 & 9.76 & 1.03 & 51.76 & \\
\hline & & Modelo ajustado & 11.02 & 18.13 & 9.31 & 1.10 & 60.44 & \\
\hline & & \% Error predictivo Roy & 33.72 & 5.04 & 8.75 & 14.04 & 7.57 & 13.83 \\
\hline & & $\%$ Error predictivo modificado & 15.22 & 5.10 & 12.95 & 7.95 & 7.92 & 9.83 \\
\hline
\end{tabular}


El tiempo de pirólisis requerido para obtener carbón más volátil depende de la tasa de transferencia de calor y de la cantidad de carbono producido; es decir, corresponde a la desvolatilización simultánea y conversión de "char" (Ahumada et al., 2016). La Tabla 3 muestra los resultados de la composición de los gases obtenidos por el modelo de Roy et al. (2009) y el modelo ajustado, estos son comparados con los datos experimentales obtenidos por Jayah et al. (2003), por lo que cada uno de estos presentan errores predictivos. Se introduce factores multiplicativos siguiendo el mismo criterio de Barman (2012) y Jarungthammachote (2008), con el fin de mejorar el rendimiento del modelo. El criterio será aplicado para diámetros de fragmentos de 3.3 a $5.5 \mathrm{~cm}$, por ejemplo se evalúa un intervalo de 0.5 a 2 para el factor multiplicativo de $\mathrm{K}_{2}$ y $\mathrm{K}_{\mathrm{R} 3}, 0.5$ a 5 para $\mathrm{r}_{\mathrm{R} 1}$ y por último de 0.5 a 0.0005 para $r_{R 2}$. Según la ecuación (14), el porcentaje de error predictivo entre el modelo actual y la referencia de la literatura (Jayah et al., 2003) se calcula de la siguiente manera (Barman, 2012):

\%Error predictivo=|(Valor experimental-valor del modelo) $/$ Valor experimental $) \mid .100 \%$

Tabla 4: Factores multiplicativos para las constantes de equilibrio $\left(K_{2}\right.$ y $\left.K_{R 3}\right)$ y velocidades cinéticas ( $r_{R_{1}}$ y $\left.r_{R 2}\right)$.

\begin{tabular}{|c|c|c|c|c|c|}
\hline $\begin{array}{c}\text { Diámetro de } \\
\text { fragment }(\mathrm{cm})\end{array}$ & $\begin{array}{c}\text { Prueba } \\
N^{\circ}\end{array}$ & $\begin{array}{c}\text { Factor para } \\
K_{2}\end{array}$ & $\begin{array}{c}\text { Factor para } \\
K_{R 3}\end{array}$ & $\begin{array}{c}\text { Factor para } \\
r_{R 1}\end{array}$ & $\begin{array}{c}\text { Factor para } \\
r_{R 2}\end{array}$ \\
\hline \multirow{3}{*}{3.3} & 1 & 1.5 & 1.5 & 1 & 1 \\
\cline { 2 - 6 } & 2 & 1.5 & 1.5 & 1 & 1 \\
\cline { 2 - 6 } & 3 & 1.5 & 1.5 & 1 & 1 \\
\hline \multirow{3}{*}{4.4} & 4 & 1.5 & 1.5 & 4 & 0.01 \\
\cline { 2 - 6 } & 5 & 1.5 & 1.5 & 4 & 0.01 \\
\cline { 2 - 6 } & 6 & 1.5 & 1.5 & 4 & 0.01 \\
\hline \multirow{3}{*}{5.5} & 7 & 1.5 & 1.5 & 3 & 0.001 \\
\cline { 2 - 6 } & 8 & 1.5 & 1.5 & 3 & 0.001 \\
\cline { 2 - 6 } & 9 & 1.5 & 1.5 & & 0.001 \\
\hline
\end{tabular}
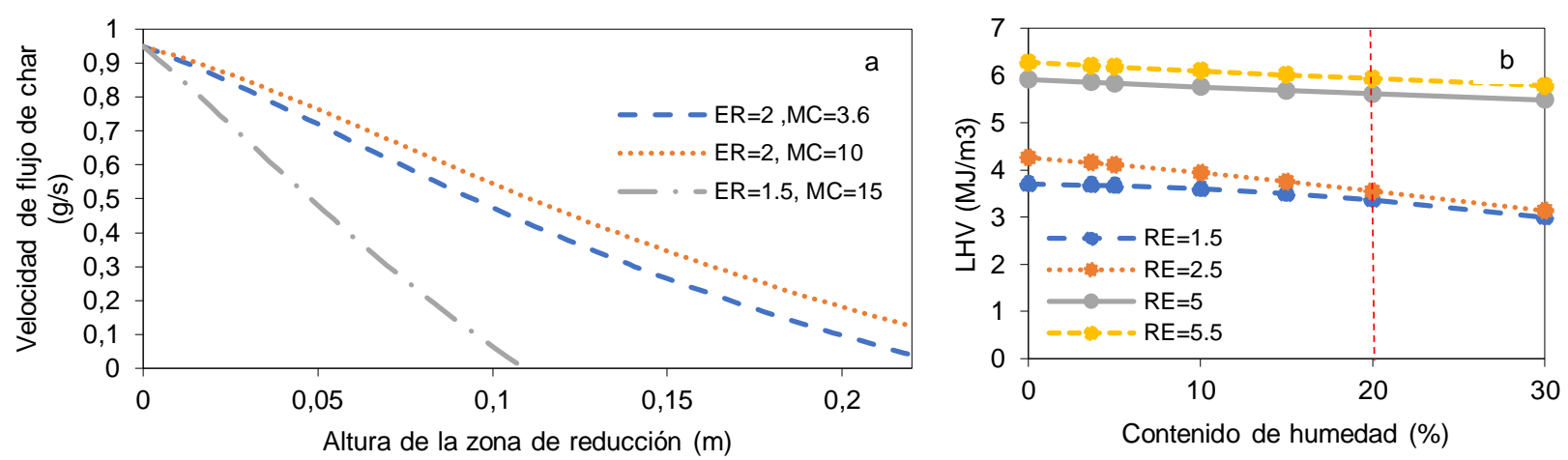

Fig. 1: (a) Disminución en la velocidad de flujo del char a lo largo de la zona de reducción y (b) Valores caloríficos del gas pobre con respecto al contenido de humedad del $R F V$ en $R E=1.5$ a $R E=5.5$.
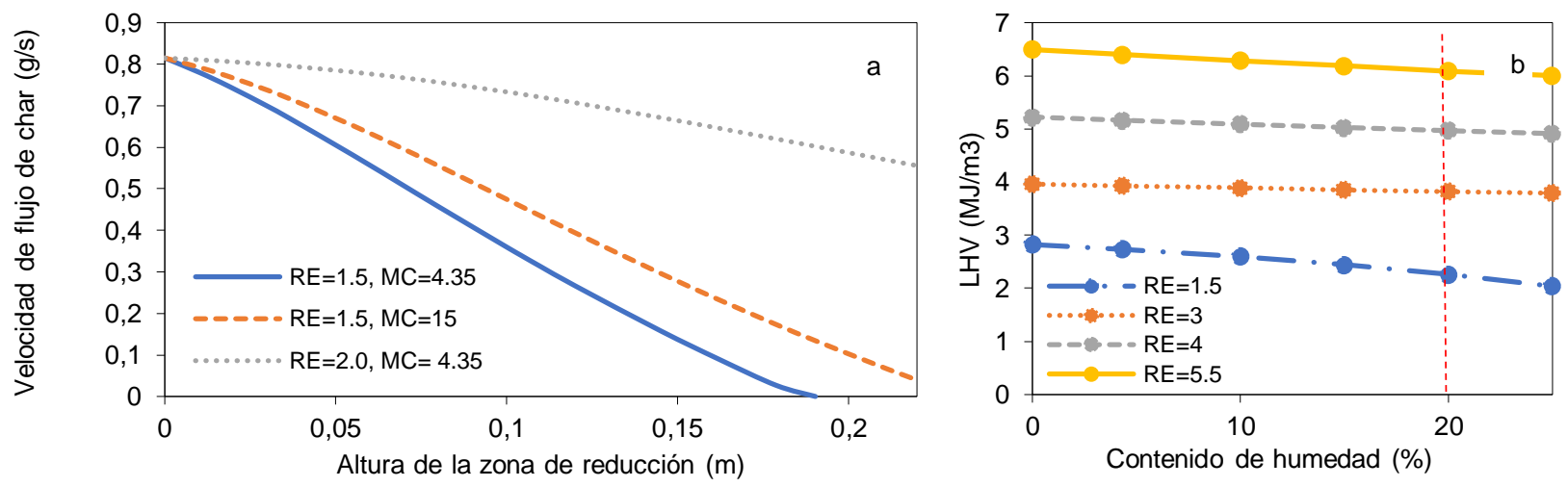

Fig. 2: (a) Disminución en la velocidad de flujo de char a lo largo de la zona de reducción y (b) Valores caloríficos del gas pobre con respecto al contenido de humedad del $C D C$ en $R E=1.5$ a $R E=5.5$.

En la Figura 1 y la Figura 2 se analiza el comportamiento de la gasificación de RFV y CDC, aplicando el modelo ajustado para fragmento de $3.3 \mathrm{~cm}$. En la Figura 1(a) y 2(a), el caudal de char tiene dos posibilidades, la primera es que el carbono fijo (char) se consuma durante todo el proceso en la zona de reducción, o la segunda, que una cierta cantidad de carbono permanezca en la salida del gasificador. Por ejemplo, en RE = 1.5 y $\mathrm{MC}=15$, durante la gasificación de RFV, el carbono se consume más rápido a lo largo de la longitud de la zona de reducción que durante la gasificación de CDC. Las Figura 1(b) y 2(b) muestran el efecto de la 
humedad en las biomasas sobre la composición del gas pobre y su valor calorífico en las diferentes relaciones de equivalencia predicho por el modelo ajustado. Según Pico et al. (2019), la relación de equivalencia tiene un fuerte efecto en el proceso de gasificación, refieren esta relación como factor preponderante en el aumento de temperatura en el proceso de gasificación y su relación inversamente proporcional al contenido de CO y $\mathrm{H}_{2}$. La calidad del gas se vuelve progresivamente más pobre en valor calorífico a medida que aumenta el contenido de humedad, considerando solo en nuestro cálculo hasta la línea roja punteada. Sin embargo, el efecto de la humedad en la reducción del valor calorífico es menor cuando el gasificador opera con una relación de equivalencia más alta.

Tanto la relación de equivalencia y la humedad de la biomasa determinan la temperatura de la zona de reducción. La elección de un máximo valor calorífico inferior del gas pobre depende de la temperatura de la zona de pirooxidación del gasificador y según Jayah et al. (2003) debe encontrarse entre $900^{\circ} \mathrm{C}$ a $1000^{\circ} \mathrm{C}$ y que el carbono fijo (char) se consuma en su totalidad en la salida de la zona de reducción del gasificador. En la tabla 5 se ha estimado la masa residual del RFV y CDC, para estimar el máximo potencial energético del gas pobre para fragmentos de $3.3 \mathrm{~cm}$ cuyos valores son 4016.4 y $2108.7 \mathrm{TJ} /$ año, respectivamente. El potencial energético del RFV y CDC disminuye para los fragmentos de $4.4 \mathrm{~cm}$ y $5.5 \mathrm{~cm}$, por lo que es necesario aumentar el tamaño dimensional del gasificador para obtener una buena calidad de gas pobre.

Tabla 5: Cantidad de masa residual seca de CDC y RFV, en el año 2017.

\begin{tabular}{|c|c|c|c|c|}
\hline Cultivos & $R_{c}(t / h a)$ & $F_{r}$ & $Y_{r s}$ & $M_{r s}(t / a n ̃ o)$ \\
\hline Cacao & 0.83 & 10.00 & 0.22 & 269208.90 \\
\hline Palma aceitera & 14.45 & 1.26 & 0.42 & 457412.50 \\
\hline
\end{tabular}

\section{CONCLUSIONES}

De acuerdo a los resultados de este estudio y de su discusión, de la comparación con resultados de otros autores se pueden extraer las siguientes conclusiones principales: 1) introducir factores multiplicativos mejoran el rendimiento del modelo de Roy para predecir la composición del gas pobre, que se ve afectada por el tamaño de los fragmentos de biomasa y por consiguiente influye mucho en el dimensionamiento del gasificador; 2) con el modelo ajustado para fragmentos de biomasa de $3.3 \mathrm{~cm}$ se obtuvo el máximo valor calorífico inferior de gas pobre tanto para RFV y CDC pero controlando la humedad, la temperatura de pirooxidación y las relaciones de equivalencia; y 3) para estimar el potencial energético de las biomasa residuales se ha planteado un modelo matemático que toma en cuenta la extracción de la energía por medio de procesos de gasificación por lo que la humedad es la variable más importante que pueden aportar los residuos de RFV y CDC; se debe tener en cuenta que el rendimiento del cultivo no tendrá un comportamiento constante; es decir, no solo dependerá de la clase de cultivo, sino de las condiciones en las que se encuentre, como la temperatura, la altura, el tipo de siembra, y el tratamiento que se da la tierra para la cosecha.

\section{AGRADECIMIENTO}

Los autores desean expresar su más sincero agradecimiento al laboratorio de la Facultad de Petroquímica por realizar el análisis de muestras y a la Facultad de Ciencias por brindarnos el sistema informático para realizar el algoritmo del programa, ambos de la Universidad Nacional de Ingeniería de Lima, Perú. Finalmente, el autor César Zavala desea agradecer al FONDECYT por el otorgamiento de una beca de doctorado (contrato $n \circ$ 207-2015-FONDECYT).

\section{NOTACIÓN}

$\begin{array}{llll}\mathrm{C}: & \text { Carbono en } \%(\mathrm{~m} / \mathrm{m}) . & \mathrm{S}: & \text { Área cultivada por año. } \\ \mathrm{H}: & \text { Hidrógeno en } \%(\mathrm{~m} / \mathrm{m}) . & \mathrm{RE}: & \text { Relación de equivalencia. } \\ \mathrm{O}: & \text { Oxígeno en } \%(\mathrm{~m} / \mathrm{m}) . & \mathrm{K}_{\mathrm{R} 1->4}: & \text { Cte. de equilibrio de la zona de reducción. } \\ \mathrm{N}: & \text { Nitrógeno en } \%(\mathrm{~m} / \mathrm{m}) . & \mathrm{K}_{1->2}: & \text { Cte. de equilibrio de la zona de pirooxidación. } \\ \mathrm{FC}: & \text { Carbono fijo en } \%(\mathrm{~m} / \mathrm{m}) . & \mathrm{r}_{\mathrm{R} 1->4}: & \text { Velocidades de reacción en la zona de reducción. } \\ \mathrm{MC}: & \text { Humedad en } \%(\mathrm{~m} / \mathrm{m}) . & \mathrm{Y}_{\mathrm{rs}}: & \text { Fracción de residuo seco. } \\ \mathrm{VM}: & \text { Materia volátil en } \%(\mathrm{~m} / \mathrm{m}) . & \mathrm{R}_{\mathrm{c}}: & \text { Rendimiento del cultivo. } \\ \mathrm{ASH}: & \text { Cenizas en } \%(\mathrm{~m} / \mathrm{m}) . & \mathrm{F}_{\mathrm{r}}: & \text { Fracción de residuo del cultivo. } \\ \mathrm{M}_{\mathrm{rr}}: & \text { Masa de residuo seco. } & \mathrm{LHV}_{\mathrm{GP}}: & \text { Valor calorífico inferior por masa de gas pobre. } \\ \mathrm{C}_{\mathrm{p}}: & \text { Capacidad calorífica estándar. } & \mathrm{g}_{\mathrm{f}}^{\circ}: & \text { Energía libre de Gibbs estándar de formación. } \\ \mathrm{h}_{\mathrm{f}}^{\circ}: & \text { Entalpia de formación estándar. } & \mathrm{T}_{\mathrm{a}}: & \text { Temperatura del aire al ingresar al gasificador. } \\ \mathrm{T}_{\mathrm{o}}: & \text { Temperatura ambiente. } & \mathrm{y}_{\mathrm{i}}: & \text { Fracción molar de la especie gaseosa. } \\ \mathrm{m}_{\mathrm{ASH}}: & \text { Masa de la ceniza. } & \mathrm{T}: & \text { Temperatura de pirooxidación. } \\ \mathrm{g}^{\circ}: & \text { Energía libre de Gibbs estándar. } & \mathrm{a}: & \text { Numero de moles de oxígeno en el aire. } \\ \mathrm{w}: & \text { Numero de moles de agua } & \mathrm{T}^{\prime}: & \text { Temperatura proveniente de la zona de reducción. }\end{array}$




\section{REFERENCIAS}

Ahumada, L.M., Verdeza, A., y otros 2 autores, Optimización de las condiciones de operación de la micro-gasificación de biomasa para producción de gas de síntesis, http://dx.doi.org/10.4067/S0718-07642016000300017, Información Tecnológica, 27(3), 179-188 (2016).

Asadieraghi, M., y Daud, W.M., In-depth investigation on thermochemical characteristics of palm oil biomasses as potential biofuel sources, https://doi.org/10.1016/j.jaap.2015.08.017, J. Anal. Appl. Pyrolysis, 115, 379-391 (2015).

ASTM, D5373-method A, Determination of carbon, hydrogen and nitrogen in analysis samples of coal and carbon in analysis samples of coal and coke, 1-11, New York, United States (2013).

ASTM, D4239, Sulfur in the analysis sample of coal and coke using high temperature tube furnace combustion methods, 1-8, United States (2004).

ASTM, D7582, Standard test methods for proximate analysis of coal and coke by macro thermogravimetric analysis, 1-9, United States (2010).

ASTM, D5865, Standard test method for gross calorific value of coal and coke, 1-14, United States (2010).

Babu, B.V., y Sheth, P.N., Modeling and simulation of reduction zone of downdraft biomass gasifier: effect of char reactivity factor, https://doi.org/10.1016/j.enconman.2005.10.032, Energy Convers. Manag., 47, 2602-2611 (2006).

Barman, N.S., Gasification of biomass in a fixed bed downdraft gasifier, https://doi.org/10.1016/j.biortech.2011.12.124, Bioresource Technology, 107,505-511 (2012).

Giltrap, D.L., McKibbin, R., y Barnes, G.R., A steady state model of gas char reactions in a downdraft gasifier, https://doi.org/10.1016/S0038-092X(03)00091-4, Solar Energy, 74, 85-91 (2003).

Hernández, J.J., Serrano, C., y otros 2 autores, Velocidad de combustión laminar del gas de gasificación calculada usando diferentes mecanismos de reacción, http://dx.doi.org/10.4067/S0718-07642004000300003, Información Tecnológica, 15(3), 19-22 (2004).

Jarungthammachote, S., y Dutta, A., Equilibrium modeling of gasification: gibbs free energy minimization approach and its application to spouted bed and spout-fluid bed gasifiers, https://doi.org/10.1016/j.enconman.2008.01.006, Energy Convers. Manag., 49, 1345-1356 (2008).

Jayah, T.H., Aye, L., y otros 2 autores, Computer simulation of a downdraft wood gasifier for tea drying, https://doi.org/10.1016/S0961-9534(03)00037-0, Biomass and Bioenergy, 25, 459-469 (2003).

Pico J.A., Soria J.A., y otros 2 autores, Modelado por técnicas de regresión de los parámetros energéticos de desempeño para gasificadores tipo downdraft, Rev. Ingeniería Energética, ISSN: 1815-5901, 40(2), 138-147 (2019).

Roy, P.C., Datta, A., y Chakraborty, N., Modeling of a downdraft biomass gasifier with finite rate kinetics in the reduction zone, https://doi.org/10.1002/er.1517, International Journal of Energy, 33, 833-851 (2009).

Serrato C.C., "Metodología para el cálculo de energía extraída a partir de la biomasa en el departamento de cundinamarca", Tesis, Universidad Distrital Francisco José de Caldas, Bogotá- Colombia (2016).

Sharma, A.K., Equilibrium and kinetic modeling of char reduction reactions in a downdraft biomass gasifier: a comparison, https://doi.org/10.1016/j.solener.2008.03.004, Solar Energy, 82, 918-928 (2008).

Thengane, S.K., Gupta, A., y Mahajani, S.M., Co-gasification of high ash biomass and high ash coal in downdraft gasifier, https://doi.org/10.1016/j.biortech.2018.11.007, Bioresource Technology, 273 159-168 (2019).

Tsai, C.H., Tsai, W.T., y otros 2 autores, Thermochemical characterization of biochar from cocoa pod husk prepared at low pyrolysis temperature, https://doi.org/10.1007/s13399-017-0259-5, Biomass Convers. Biorefin., 8, 237-243 (2018).

Torres C., Chaves M., y otros 2 autores, Evaluación de la incidencia de pellets y astillas de madera en el desempeño de un gasificador tipo "downdraft", https://doi.org/10.18845/rfmk.v15i1.3847, 15(1), 25-36 (2018).

Valadez, L., Ortiz, A., y otros 3 autores, Valorization of cacao pod husk through supercritical fluid extraction of phenolic compounds, https://doi.org/10.1016/j.supflu.2017.09.011, Journal of Supercritical Fluids,131, 99-105 (2018).

Varnero, M.T., Quiroz, M.S., y Álvarez, C.H., Utilización de residuos forestales lignocelulósicos para producción del hongo ostra (Pleurotus ostreatus), http://dx.doi.org/10.4067/S0718-07642010000200003, Información Tecnológica, 21(2), 13-20 (2010).

Wang, Y., y Kinoshita, C.M., Kinetic model of biomass gasification, https://doi.org/10.1016/0038-092X(93)90037-O, Solar Energy, 51, 19-25 (1993).

Waldheim, L., Heating value of gases from biomass gasification, Report, IEA Bioenergy (2001).

Yan, T.G., Kong, L.X., y otros 3 autores, Thermomechanical analysis of coal ash fusion behavior, https://doi.org/10.1016/j.ces.2016.03.016, Chemical Engineering Science, 147, 74-82 (2016).

Yiin, C.L., Yusup, S., y otro 4 autores, Thermogravimetric analysis and kinetic modeling of low transition temperature mixtures pretreated oil palm empty fruit bunch for possible maximum yield of pyrolysis oil, https://doi.org/10.1016/j.biortech.2018.01.132, Bioresource Technology, 255, 189-197 (2018). 